\title{
ENFOQUES DE APRENDIZAJE DEL ALUMNADO UNIVERSITARIO EN FUNCIÓN DE LOS CONTEXTOS DE AULA Y CURSO
}

\author{
Javier Goikoetxea Piérola \\ Iker Ros Martínez de Lahidalga \\ Karmele Buján Vidales \\ Universidad del País Vasco
}

\begin{abstract}
RESUMEN: El objetivo del estudio ha sido analizar la influencia de la percepción del contexto de enseñanza y aprendizaje de aula y la de contexto de curso en los enfoques de aprendizaje de los estudiantes universitarios por titulaciones, campus y cursos. La muestra está compuesta por 524 estudiantes de primer y segundo curso de Magisterio, Enfermería y Escuelas Politécnicas de Álava y Guipúzcoa. Las escalas utilizadas fueron: el R-SPQ-2F (Biggs, Kember y Leung, 2001) para medir los enfoques de aprendizaje, y las subescalas de Estrategias Docentes y de Aprendizaje (GEDA) (San Fabián, Argós, Goikoetxea, 2009; Goikoetxea, Aramendi, Bujan, Rekalde y Ros, 2010) para medir los contextos de aula y de curso. El estudio sirvió para confirmar la importancia de dos variables contextuales poco estudiadas hasta ahora, el contexto del aula y el contexto de curso, y sus relaciones con el enfoque de aprendizaje de los estudiantes universitarios. El contexto del aula es una variable que se diferencia entre los grados y también se relaciona con variaciones significativas en los enfoques profundos y superficiales de los estudiantes. El contexto de curso se manifiesta como una variable específica de grado: las diferencias más significativas para esta variable se dan entre los mismos estudiantes cuando están en el primer o segundo año de la carrera.
\end{abstract}

PALABRAS CLAVE: Percepción del contexto de enseñanza y aprendizaje de aula, percepción de contexto de curso, enfoques de aprendizaje, estudiantes universitarios.

\section{HIGHER EDUCATION STUDENT'S APPROACHES TO LEARNING ACCORDING TO THEIR PERCEPTION OF THE CONTEXT OF COURSE AND CLASSROOM}

\footnotetext{
ABSTRACT: The objective of this research was to analyze the relationship between the perception of teaching and learning classroom contexts and
} 
perception of course context with approaches to learning for college students. These influences are contrasted by degree, campus and courses. The sample is composed by 524 students of first and second year of Infant and Primary Education, Nursing and Polytechnics of Alava and Guipuzcoa campus. Used scales are the R-SPQ-2F (Biggs, Kember y Leung, 2001) to measure the student's approaches to learning and the subscales of general Questionnaire of Teaching's and Learning's Strategies (TS-LSQ) (San Fabián, Argós, Goikoetxea, 2009; Goikoetxea, Aramendi, Bujan, Rekalde y Ros, 2010) to measure the classroom's context and current context. The study served to confirm the importance of two contextual variables poorly studied until now, the classroom context and the context of course, and its relations with the learning approach of university students. The classroom context is a variable that differentiates between degrees and also is related to significant variations with deep and surface approaches of students. The variable context of course is manifested as a specific variable of degree: the most significant differences for this variable are between the same students when they are in first or second year of career.

KEYWORDS: Perception of teaching and learning classroom's context, perception of context of course, approaches to learning, higher education students.

Recibido: 13/10/2012

Aceptado: 07/02/2013

\section{INTRODUCCIÓN}

Los enfoques de aprendizaje profundo y superficial son un modo de describir de forma continua la estrategia cognitiva general (motivación más estrategia) ante una situación compleja de enseñanza y aprendizaje que ha sido objeto de estudio de una larga tradición investigadora (la investigación fenomenográfica del aprendizaje, Marton y Säljö, 1976). Los enfoques de aprendizaje, según Biggs (1988, 2001), se caracterizan por la influencia del proceso metacognitivo como elemento mediador entre la intención o motivo del estudiante y la estrategia de aprendizaje utilizada para estudiar. Esta variable ha sido tradicionalmente objeto de estudio de las investigaciones inspiradas en el modelo SAL, Students Approach to Learning (Biggs, 1978, 1987, 2001 y Prosser, Trigwell, Hazel, y Gallagher, 1994; Ramsden, 2003).

Desde hace tiempo se viene reconociendo que la variable de proceso enfoques de aprendizaje no presenta estadísticamente resultados claros a la hora de describir la disposición estratégica de los estudiantes y sus cambios ante la modificación de los contextos de enseñanza-aprendizaje. Sin embargo, se acepta que esta variable sirve como un buen indicador general de los cambios en la motivación y estrategia de aprendizaje del alumnado y como un recurso para la orientación de la innovación metodológica de los procesos de enseñanza y aprendizaje (Berben, De la Fuente, Justicia y Pichardo, 2005). 
Las variables contexto de enseñanza y aprendizaje y el contexto de curso han sido estudiadas por Ramsden, 1991, 1992, 2003; Weinstein y Mayer, 1986; Vermunt, 2003; y Prosser y Trigwell, 1997, entre otros. Los estudios desarrollados tradicionalmente desde la teoría de los enfoques de aprendizaje de los estudiantes (SAL) se han centrado en las variables del contexto de enseñanza-aprendizaje como métodos de evaluación, métodos de enseñanza, características del profesorado y rendimiento académico y comparten el supuesto de que los enfoques de aprendizaje dependen más de estas variables contextuales que de las variables individuales, sin excluir la influencia de estas últimas (Biggs, 1996, 2001; Biggs, Kember y Leung, 2001). La mayoría de estos estudios argumentan que existe una relación positiva entre las metodologías de enseñanza centradas en la transmisión del contenido y los enfoques superficiales adoptados por los estudiantes, así como entre las metodologías de enseñanza centradas en el alumnado y los enfoques profundos de éstos (Buendía y Olmedo, 2000; Entwistle y Tait, 1990; Kember y Gow, 1994; Trigwell, Prosser y Waterhouse, 1999; Prosser y Trigwell, 1999). Estudios recientes en el contexto español establecen parecidas relaciones entre metodología de enseñanza (aprendizaje autónomo) y aprendizaje autorregulado (De la Fuente, 2011); estilo docente del profesorado (centrado en el estudiante) y desarrollo de enfoques y estrategias cognitivas más profundas (Gargallo, Fernández, Garfella y Pérez, 2010); relación entre la memoria y el proceso de memorización como estrategia de aprendizaje en la adquisición de la lengua (Corpas, 2008); uso de metodologías activas de enseñanza (Alcoba, 2012) y enfoques más profundos de aprendizaje (Palazón, Gómez, Gómez y Pérez, 2011).

En este trabajo se pretende describir el comportamiento de dos variables contextuales complejas y poco estudiadas (Ramsdem, 1991; Entwistle y Ramsdem, 1983): 1) la percepción por los estudiantes universitarios de los contextos de enseñanza y aprendizaje del conjunto de profesoras y profesores de curso, que se denomina en este estudio contexto de aula (CA); y 2) la percepción del contexto de curso (CC), entendido como los niveles de coordinación y colaboración entre las profesoras y los profesores de curso para establecer estándares comunes de carga de trabajo de los estudiantes, metodologías de enseñanza y aprendizaje parecidos, y criterios de evaluación compartidos. Y 3) en tercer lugar, describir los enfoques de aprendizaje de los estudiantes. Las dos variables contextuales se definen a partir de los trabajos de Ramsdem y Entwistle (Ramsdem, 1991; Entwistle y Ramsdem, 1983) y otros (Ramsden, Prosser, Trigwell y Martin, 1997; Prosser y Trigwell, 1999).

Los objetivos de esta investigación son principalmente: 1) Identificar diferencias por titulaciones (de Grado y Diplomatura o Licenciatura) y por cursos (primero y segundo) en las relaciones entre las tres variables. 2) Describir en diversos momentos las diferencias entre estas variables en curso primero y curso segundo (solo posible en el caso de las antiguas titulaciones). Y 3) Establecer las relaciones generales entre las variables de contexto: a) percepción por los estudiantes del contexto de enseñanza y aprendizaje del conjunto de profesores y profesoras de curso (contexto de aula), b) percepción por los estudiantes del contexto de curso, y c) la variable de Proceso: los enfoques de aprendizaje de los estudiantes que puedan derivarse del conjunto de la muestra. 


\section{Método}

\section{Participantes}

Todos los alumnos objeto de estudio pertenecen a las titulaciones de Grado de Maestro en Educación Infantil y Primaria, Enfermería y Escuelas Politécnicas de los campus de Álava y Guipúzcoa de la Universidad del País Vasco conformando un total de 524 sujetos.

Tabla 1. Distribución de los estudiantes de la Universidad del País Vasco por campus y titulación

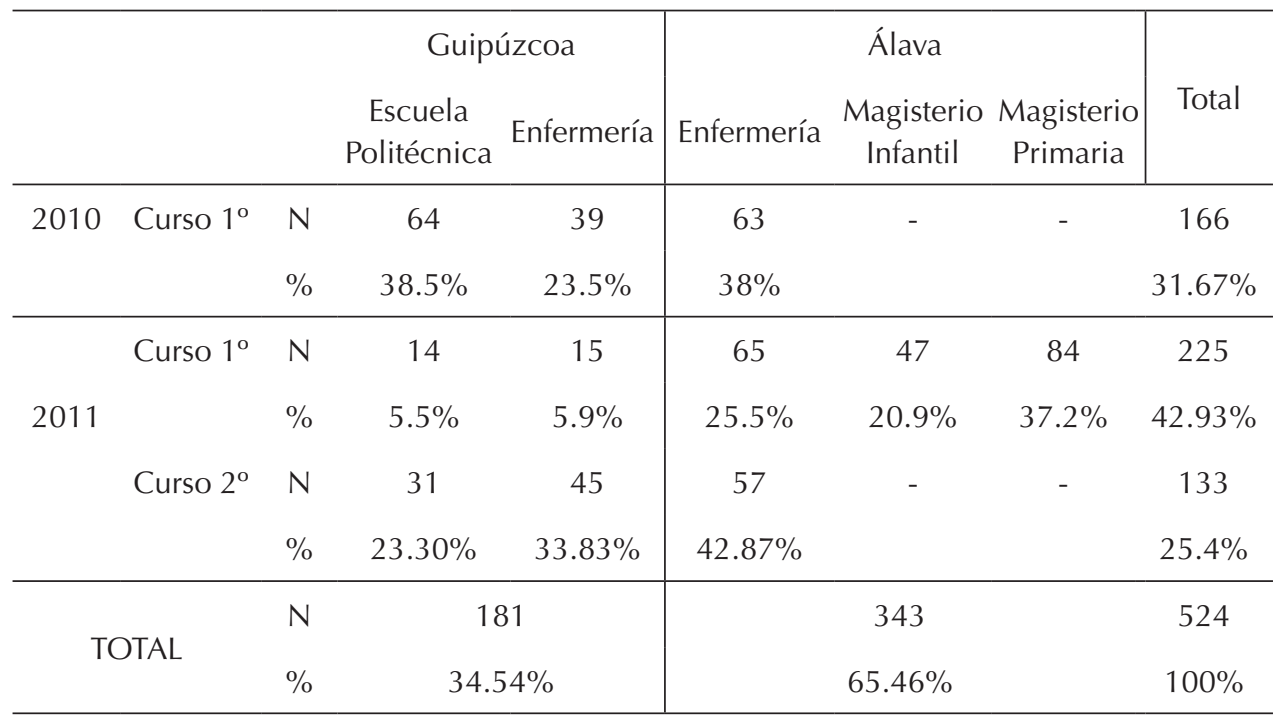

\section{Variables e instrumentos de medida}

En el diseño del proyecto, las dimensiones y variables se integran en un cuestionario que pretende contribuir al logro de los tres objetivos del estudio anteriormente reseñados: de una parte, recoger información a nivel general, de titulación y a nivel longitudinal sobre la percepción del contexto de enseñanza y aprendizaje del aula y del contexto de curso y, de otra, relacionarlo con los enfoques de aprendizaje de los estudiantes. De entre todas las variables contempladas en el cuestionario, se relacionan, a continuación, aquellas en las que se ha centrado este trabajo.

Para medir la percepción del contexto de enseñanza-aprendizaje del aula y del contexto de curso y los enfoques de aprendizaje de los estudiantes se ha elaborado el cuestionario General de Estrategias Docentes y de Aprendizaje (GEDA) (San Fabian, Argos, Goikoetxea, 2009; Goikoetxea et al., 2010). Para elaborarlo se han tenido en cuenta el cuestionario CEVEADEPU (Cuestionario de metodología de enseñanza y evaluación de los profesores universitarios, de Gargallo et al., 2010). Otro de los cuestionarios utilizados ha sido el R-SPQ-2F de Biggs et al. (2001) para recoger los ítems sobre el pensamiento estratégico. 
Cuadro 1. Percepción por los estudiantes del contexto de enseñanza y aprendizaje del conjunto de profesores de curso (Contexto de aula, CA)

\begin{tabular}{|c|c|c|}
\hline \multirow{4}{*}{$\begin{array}{l}\text { D.1) Percepción } \\
\text { del docente } \\
\text { (estilo docente } \\
\text { y relacional) (20 } \\
\text { ítems) }\end{array}$} & \multirow{3}{*}{$\begin{array}{l}\text { 1.1) Percepción } \\
\text { metodología } \\
\text { docente del } \\
\text { profesorado ( } 14 \\
\text { ítems) }\end{array}$} & $\begin{array}{l}\text { 1.1.1) Adecuación de los contenidos al } \\
\text { conocimiento previo de los estudiantes y } \\
\text { significatividad interna (Prosser y trigwell,1999) } \\
\text { (5 ítems) }\end{array}$ \\
\hline & & $\begin{array}{l}\text { 1.1.2) Clima de participación, comunicación } \\
\text { profesor/a-estudiantes ( } 4 \text { ítems) }\end{array}$ \\
\hline & & $\begin{array}{l}\text { 1.1.3) Estilo docente (centrado en la transmisión / } \\
\text { centrado en el aprendizaje) ( } 2 \text { ítems) }\end{array}$ \\
\hline & \multicolumn{2}{|c|}{$\begin{array}{l}\text { 1.2) Percepción Prioridades del profesorado (objetivos de } \\
\text { aprendizaje: adquisición de contenidos, destrezas, capacidades, } \\
\text { resolución problemas prácticos, competencias profesionales, } \\
\text { actitudes y valores) (6 ítems) }\end{array}$} \\
\hline \multirow{3}{*}{$\begin{array}{l}\text { D.2) Percepción } \\
\text { de la práctica } \\
\text { docente del } \\
\text { profesorado ( } 37 \\
\text { ítems) }\end{array}$} & \multicolumn{2}{|c|}{ 2.1) Nivel de uso de distintas metodologías de enseñanza (14 ítems) } \\
\hline & \multicolumn{2}{|c|}{ 2.2) Nivel de uso de diferentes recursos materiales (10 ítems) } \\
\hline & \multicolumn{2}{|c|}{ 2.3) Nivel de uso de distintos tipos de evaluación (13 ítems) } \\
\hline
\end{tabular}

El cuestionario completo consta de 198 ítems divididos en 3 apartados: características individuales, percepción del contexto de enseñanza, y planteamiento estratégico. Las tres escalas de las variables estudiadas (contexto de aula, contexto de curso, enfoques de aprendizaje) se integran en el cuestionario general. Las respuestas corresponden a una escala de tipo Likert con valores comprendidos entre el 1 y el 5 ( 1 muy en desacuerdo, 2 en desacuerdo, 3 término medio, 4 de acuerdo y 5 muy de acuerdo). No son objetivo de este trabajo las características individuales que serán trabajadas en posteriores aportaciones. La variable percepción por los estudiantes del contexto de enseñanza y aprendizaje del conjunto de profesores y profesoras de curso, o el contexto de aula, se compone de dos subescalas (ver cuadro 1) recogidas en el cuestionario genérico de Estrategias de Enseñanza y Aprendizaje, GEDA (San Fabian, Argós, Goikoetxea, 2009; Goikoetxea et al., 2010). La percepción del contexto de aula obtiene un alpha de Crombach de $\alpha=.862$ (50 ítems).

La variable percepción del contexto de curso se mide a través de una subescala del cuestionario GEDA que se compone de seis ítems: coordinación metodológica, niveles de exigencia, conocimiento de profesor/a coordinador/a de curso, exceso de contenidos en la mayoría de asignaturas, diferencias de dedicación y esfuerzo que exigen las asignaturas, acumulación de trabajo en determinados momentos del curso. La percepción del contexto de curso obtiene un alpha de Crombach de $\alpha=.528$ (6 ítems).

El planteamiento estratégico, la información de los estudiantes sobre los enfoques de aprendizaje se recogió mediante el cuestionario RSPQ-2F de Biggs et al. (2001) que integra los ítems en las subescalas de motivación y estrategia para elaborar, a partir de ellas, las escalas que definen los enfoques de aprendizaje profundo y superficial. El coeficiente alpha de Cronbach de las escalas de los enfoques, obtiene un alpha de $\alpha=.772$ (10 ítems) para el enfoque profundo y $\alpha=.758$ para el enfoque 
superficial. Estos índices se sitúan en magnitudes similares a las referidas por Hidalgo (2010) con una muestra española y con las mismas discrepancias respecto a las encontradas por Biggs et al. (2001).

Las subescalas se describen a continuación: 1) En primer lugar, se considera la motivación con dos niveles diferenciados. La motivación profunda (Deep Motivation - DM): motivación e interés intrínseco del estudiante por la tarea, sin preocuparse por el esfuerzo requerido y asistencia a las clases con preguntas preparadas. Y la motivación superficial (Superficial Motivation - SM) o motivación extrínseca a la tarea en la que el estudiante aprende intentando salir del paso con el menor esfuerzo posible. 2) En segundo lugar, se contempla la estrategia con otros dos niveles. La estrategia profunda (Deep Strategy - DS): el estudiante trata de comprender el significado de los temas y dedica tiempo extra para completarlos. Y la estrategia superficial (Superficial Strategy - SS): el estudiante se centra exclusivamente en lo requerido para aprobar aprendiendo los contenidos de forma mecánica y repetitiva para reproducirlos sin aportación personal.

A continuación se exponen las escalas que, como se ha indicado, surgen de la adición de las distintas subescalas. Así aparece, por un lado, el enfoque profundo (Deep Approach - DA) como adición de DM y DS y que caracteriza al alumno por tener motivación intrínseca, preocupación por aprender, dedicación de tiempo extra... Y, por otro, el enfoque superficial (Superficial Approach - SA) como la suma de SM y SS y que define al estudiante con motivación extrínseca, cumplimiento de tareas con el menor esfuerzo, estudio repetitivo...

\section{Procedimiento}

En el estudio se ha utilizado la información proporcionada por los estudiantes y recogida mediante cuestionarios de opinión Este trabajo se integra en otro más amplio en el que se analizan las variables que determinan las estrategias y motivaciones de cara a los estudios universitarios de los profesores y estudiantes de las universidades de Cantabria, Oviedo y el País Vasco (San Fabián et al., 2009) y se sustenta en una metodología mixta en cuanto a métodos (cuestionario, entrevistas y grupos de discusión) y a fuentes (estudiantes, profesores y equipos directivos). Se trata de un diseño de corte longitudinal con recogida de información mediante los instrumentos indicados más adelante, en tres momentos (inicial, intermedio final) a los mismos estudiantes. Mediante el contraste de métodos y de fuentes se podrá determinar la evolución ínter e intrasujetos de los temas objeto de estudio (enfoques de aprendizaje y percepción de contexto).

Para este trabajo, se contó con la participación voluntaria de los estudiantes de los centros analizados en los campus de San Sebastián y Vitoria. Se contactó con los directores de los centros escolares donde estudiaban, solicitando permiso para pasar el cuestionario y se acordó la fecha para recoger la información. El cuestionario se completó de forma colectiva en horario de clase con la ayuda de los profesores del propio centro, y el pase de la prueba fue realizado en todos los casos por las mismas personas, quienes explicaban la prueba y aclaraban todas las posibles dudas, comentando también que se respetaría en todo momento el anonimato de los participantes. La recogida de datos se realizó durante el primer cuatrimestre del año 2010 y del 2011. La intervención en el centro ha incluido visitas explicativas, creación de comisión de trabajo, adaptación de los instrumentos, apoyo durante el estudio y devolución de los resultados. 


\section{Resultados}

Se hará un tratamiento estadístico de los datos con el programa SPSS15. El análisis de las características psicométricas de nuestros datos arroja resultados similares a los obtenidos en otras investigaciones relativas a los enfoques de aprendizaje (Biggs, 2001; De la Fuente et al., 2008).

En orden a clarificar el primer objetivo de este trabajo, e identificar diferencias por titulaciones (de Grado y Diplomatura o Licenciatura) y por cursos (primero y segundo) en las relaciones entre las tres variables, se procederá a correlacionar las variables a observar con los factores mencionados buscando relaciones significativas ( $r$ de Pearson).

Tabla 2. Análisis post-hoc de la diferencia de medias de los enfoques de aprendizaje y la percepción del contexto de enseñanza-aprendizaje de aula y del contexto de curso entre las diferentes carreras

\begin{tabular}{ccc|cc}
\hline & \multicolumn{2}{c|}{ CONTEXTOS } & \multicolumn{2}{c}{ ENFOQUES } \\
& CA & CC & DA & SA \\
\hline Titulación & $.096^{*}$ & $-.088^{*}$ & -.060 & $.186^{* *}$ \\
Curso & $-.289^{* *}$ & $-.312^{* *}$ & -.087 & $.096^{*}$ \\
Campus & .055 & .008 & .019 & -.001 \\
\hline
\end{tabular}

* Nivel de significación: .05 ** Nivel de significación: .01

LEYENDA: $C A=$ Contexto de aula; $C C=$ Contexto de curso; $D A=$ Enfoque profundo; $S A=$ Enfoque superficial

Las puntuaciones de las correlaciones pueden parecer bajas en comparación con otros estudios pero hay que tener en cuenta la naturaleza compleja de las variables contexto de aula y contexto de curso y la composición de la muestra: estudiantes de primero y segundo curso.

Posteriormente y tras comprobar la normalidad de la muestra se realizó un contraste de medias con la prueba $t$ de student cuando se trataba de variables dicotómicas (campus y curso) y el contraste de medias con la prueba de la ANOVA obteniendo la $F$ de Fisher y posteriores pruebas post-hoc de contraste (HSD de Tukey) para comprobar la diferencia específica entre titulaciones y entre centros.

Tabla 3. Análisis post-hoc de la diferencia de medias de los enfoques de aprendizaje y la percepción del contexto de aula y curso entre las diferentes carreras

\begin{tabular}{ccccccc}
\hline & & & CA & CC & DA & SA \\
\hline$F$ & & & $11.408^{* *}$ & 2.249 & $3.679^{*}$ & $16.804^{* *}$ \\
HSD de & Enfermería & Politécnica & $.087^{*}$ & .020 & $1.803^{*}$ & $-3.325^{* *}$ \\
$\begin{array}{c}\text { Tukey } \\
\text { (Dif. }\end{array}$ & Magisterio & Enfermería & $.090^{* *}$ & -.118 & -.583 & $2.099^{* *}$ \\
Medias) & & Politécnica & $.177^{* *}$ & -.097 & 1.219 & -1.225 \\
\hline
\end{tabular}

* Nivel de significación: .05 ** Nivel de significación: .01

LEYENDA: $C A=$ Contexto de aula; $C C=$ Contexto de curso; $D A=$ Enfoque profundo; $S A=$ Enfoque superficial 
Lo que nos dicen estos datos es que las variables se están comportando básicamente como se esperaba: el contexto de aula es una variable que sirve para establecer diferencias entre titulaciones; pudiera parecer que hay que desechar la variable contexto de curso por no ser capaz de establecer esas diferencias. Sin embargo, estos datos están sugiriendo que la variable de contexto de curso es específicamente una variable del nivel de titulación y no del nivel interinstitucional o de intertitulaciones. Finalmente, la variable enfoques de aprendizaje se comporta de acuerdo con su naturaleza, como indicador indirecto de los cambios en los contextos de aula y de curso.

Tabla 4. Análisis post-hoc de la diferencia de medias de los enfoques de aprendizaje y la percepción del contexto de aula y curso entre las diferentes carreras

\begin{tabular}{|c|c|c|c|c|c|c|}
\hline & & & $\mathrm{CA}$ & $\mathrm{CC}$ & DA & SA \\
\hline \multirow[t]{3}{*}{$F$} & & & $11.689 * *$ & $53.015^{* *}$ & $1.910^{*}$ & $5.358^{* *}$ \\
\hline & $1^{\circ}$ PAEV 10 & $1^{\circ}$ PAED10 & -.142 & $.373^{* *}$ & -1.443 & .209 \\
\hline & $1^{\circ}$ PAEV 10 & $1^{\circ} \mathrm{GEV} 11$ & .089 & $1.042^{* *}$ & -.819 & -1.287 \\
\hline \multirow{4}{*}{$\begin{array}{l}\text { HSD de } \\
\text { Tukey (Dif. } \\
\text { Medias) }\end{array}$} & $1^{\circ}$ GEV 11 & $1^{\circ} \mathrm{GED} 11$ & -.132 & -.077 & .078 & 1.841 \\
\hline & $2^{\circ}$ PAEV11 & $2^{\circ}$ PAED11 & $-.387^{* *}$ & -.039 & -3.339 & $-3.933^{*}$ \\
\hline & $1^{\circ}$ MIV11 & $1^{\circ} \mathrm{MPV} 11$ & -.020 & $-.328^{* *}$ & -1.015 & -2.157 \\
\hline & $1^{\circ}$ PD 2010 & 10 PD 2011 & -.135 & $.825^{* *}$ & -1.705 & 1.113 \\
\hline
\end{tabular}

* Nivel de significación: .05** Nivel de significación: .01

LEYENDA: $1^{\circ}$ PAEV11=primero, plan antiguo, Enfermería, Vitoria, 2011; $2^{\circ}$ PAEV11=segundo, plan antiguo, Enfermería, Vitoria, 2011; $1^{\circ}$ MIV11=primero, magisterio, infantil, Vitoria, 2011; $1^{\circ}$ PD 2010=primero, Politécnica, Donostia, 2010; $1^{\circ}$ PAED 2010=primero, plan antiguo, enfermería, Donostia, 2010; $1^{\circ}$ GEV 2011=primero, Grado, Enfermería, Vitoria, 2011; 1GED 2010= primero, Grado, Enfermería, Donostia; $2^{\circ}$ PAED $2011=$ segundo, Plan Antiguo, Enfermería, Donostia,2011; $1^{\circ}$ MPV $2011=$ primero, Magisterio, Primaria, 2011; $1^{\circ}$ PD 2011=primero, Politécnica, Donostia, 2011; CA=Contexto de aula; CC=Contexto de curso; $\mathrm{DA}=$ Enfoque profundo; $\mathrm{SA}=$ Enfoque superficial

Estos datos sugieren que la variable contexto de curso varía entre titulaciones antiguas y de grado, en el caso de Enfermería de Vitoria y Politécnica de San Sebastián, y entre mismas titulaciones de diferentes centros, como es el caso de los cursos primero de plan antiguo de Enfermería de Vitoria y de San Sebastián. Las mismas titulaciones y centros manifiestan diferencias significativas en el enfoque superficial en los cursos segundo, lo que sugiere que el enfoque superficial de estas titulaciones y centros puede relacionarse con el contexto de curso. Así, la variable contexto de curso, además de indicarnos diferencias entre titulaciones y centros, puede relacionarse con las diferencias de los centros y titulaciones en el enfoque superficial. Estos datos refuerzan la argumentación anterior acerca de que la variable contexto de curso es fundamentalmente de titulación y que será mejor percibida por los estudiantes a medida que vayan pasando de cursos. Esto no impide 
establecer comparaciones con los modos de percibir esta variable por estudiantes de diferentes titulaciones.

Tabla 5. Análisis de la diferencia de medias entre los enfoques y estrategias de aprendizaje y entre la percepción del contexto de aula y la percepción de curso

\begin{tabular}{lcccc}
\hline & CA & CC & DA & SA \\
$\begin{array}{l}\text { Enfermería VI 2010 } \\
\text { Enfermería VI 2011 }\end{array}$ & $5.827^{* *}$ & $16.034^{* *}$ & .599 & -1.252 \\
$\begin{array}{l}\text { Enfermería SS 2010 } \\
\text { Enfermería SS 2011 }\end{array}$ & .894 & $8.808^{* *}$ & -.935 & 1.283 \\
$\quad \begin{array}{l}\text { Politécnica SS 2010 } \\
\text { Politécnica SS 2011 }\end{array}$ & -1.430 & $10.460^{* *}$ & .149 & -1.852 \\
\hline ** Nivel de significación: .01 & & & \\
LEYENDA: CA=Contexto de aula; CC=Contexto de curso; DA=Enfoque profundo; SA=Enfoque superficial
\end{tabular}

Los datos indican que solamente se da una variación significativa en la percepción del contexto de aula entre el curso primero y segundo de enfermería de VitoriaGasteiz. En cuanto la variable contexto de curso, el nivel de coordinación metodológica y de carga de trabajo entre asignaturas, apenas es percibida por los estudiantes en el primer curso de carrera pero va haciéndose más evidente a medida que se coge experiencia académica (curso segundo). Los datos indican igualmente que las diferencias de percepción del contexto de curso entre los mismos estudiantes cuando están en primero o en segundo de carrera varían entre titulaciones. Y que estas diferencias de percepción del contexto de curso parecen ser de naturaleza distinta en cada titulación pues tienen efectos muy diversos sobre el enfoque profundo y el enfoque superficial. Hecho que refuerza la idea de considerar la variable contexto de curso como variable específica de titulación.

Tabla 6. Análisis correlacional de los enfoques y estrategias de aprendizaje con la percepción del contexto de aula y la percepción de contexto de curso de los estudiantes de la Universidad del País Vasco (2010-2011)

\begin{tabular}{cccc}
\hline & $\begin{array}{c}\text { Enfoque } \\
\text { profundo }\end{array}$ & $\begin{array}{c}\text { Enfoque } \\
\text { superficial }\end{array}$ & $\begin{array}{c}\text { Contexto } \\
\text { de aula }\end{array}$ \\
\hline Contexto de aula & $.427^{* *}$ & $-.203^{* *}$ & - \\
Contexto de curso & .030 & -.027 & $.179^{* *}$ \\
\hline
\end{tabular}

** Nivel de significación: .01

Los datos obtenidos también confirman la hipótesis de partida de que la variable contexto de enseñanza y aprendizaje del conjunto de docentes del aula es una variable contextual por derecho propio y que se relaciona especialmente, de forma significativa y positiva, con las variaciones en el enfoque profundo de los estudiantes $\left(r=.427^{* *}\right)$ y, de forma significativa y negativa, con las variaciones en el enfoque 
superficial $\left(r=-.203^{* *}\right)$. Estos datos hablan también de la calidad de los contextos de aula de estas cuatro titulaciones en sus primeros y segundos cursos: la percepción de estos contextos por los estudiantes se relaciona con una orientación moderada a adoptar un enfoque profundo en el aprendizaje $y$, en menor medida, a desestimar la utilización del enfoque superficial. Y, si bien, el porcentaje de varianza de los enfoques de aprendizaje explicado por la variable contexto de aula (18\% del enfoque profundo y $4 \%$ del enfoque superficial) no es muy alto, este dato puede explicarse por la naturaleza de la propia variable, centrada en los contextos de enseñanza y aprendizaje del conjunto de docentes de curso y no en metodologías específicas de cada docente concreto, y de la constitución de la muestra: primeros y segundos cursos de carrera.

Si se tiene en cuenta las dimensiones específicas de la variable contexto de aula, los estudiantes encuentran mayor motivación profunda inspirándose en las prioridades de los docentes $\left(r=-.361^{* *}\right)$ y, en segundo lugar, en su metodología $\left(r=-.307^{* *}\right.$; $\left.r=-.282^{* *}\right)$ y deciden la estrategia profunda de aprendizaje fijándose primero en la metodología del docente $\left(r=-.287^{* *} ; r=-.245^{* *}\right)$ y, en segundo lugar, en las prioridades del docente $\left(r=-.243^{* *}\right)$. Los tipos de evaluación utilizada por el docente es la tercera fuente de inspiración para adoptar una motivación profunda $\left(r=-.194^{* *}\right)$ y una estrategia profunda de aprendizaje $\left(r=-.205^{* *}\right)$.

La motivación superficial de los estudiantes se relaciona, en cambio, con las prioridades del docente $\left(r=-.176^{* *}\right)$ y con las metodologías docentes utilizadas $(r=-$ $\left..138^{* *}\right)$. Y la estrategia superficial del alumnado se relaciona exclusivamente con las metodologías docentes utilizadas $(r=-.122 * *)$.

Estos datos son consistentes con los resultados de otros estudios (Ramsdem et al., 1997; Prosser y Trigwell, 1999) que reconocen la importancia de los objetivos claros y de la buena enseñanza, ésta como prioritaria, y de la evaluación, como variables que se relacionan con los enfoques de aprendizaje de los estudiantes. Por su parte, la metodología de evaluación es una variable contextual que ha sido tradicionalmente tenida en cuenta en los estudios del enfoque de aprendizaje de los estudiantes SAL (Entwistle y Tait, 1990; Biggs, 2001).

\section{DısCUSıÓn}

El estudio ha servido para confirmar la importancia de dos nuevas variables contextuales poco estudiadas hasta ahora, el contexto de aula y el contexto de curso, y sus relaciones con el enfoque de aprendizaje del alumnado. El contexto de aula, como el contexto de enseñanza y aprendizaje del conjunto de docentes de un grupocurso, es una variable que establece diferencias entre titulaciones, como se señala en tabla 3: los contextos de aula de la titulación de Magisterio difieren de forma significativa de los contextos de aula de la titulación Politécnica $\left(.177^{* *}\right)$ y de la titulación de Enfermería $\left(0,90^{* *}\right)$, la diferencia entre Politécnica y Enfermería es menor y moderadamente significativa $\left(0,87^{*}\right)$. A su vez, el contexto de aula se relaciona con variaciones significativas en los enfoques profundos $\left(r=.427^{* *}\right)$ y superficiales $\left(r=-.203^{* *}\right)$ de aprendizaje de los estudiantes de primer y segundo curso de las cuatro titulaciones estudiadas. El análisis de las dimensiones específicas de esta variable sugiere que las prioridades de los docentes, las metodologías de enseñanza y las 
tipologías de evaluación se relacionan con la adopción de unos enfoques u otros por parte de los estudiantes.

Los resultados parecen indicar que los estudiantes que adoptan un enfoque profundo en el aprendizaje se fijan especialmente en variables contextuales que ayudan a captar el sentido y el significado global de la actividad de enseñanzaaprendizaje, tales como la impresión global que les causa el docente (su estilo docente y relacional), su metodología general de enseñanza (que incluye aspectos como la adecuación de los contenidos al conocimiento previo de los estudiantes y la significatividad interna de los contenidos, el clima de participación y comunicación entre docente y estudiantes, y su estilo docente centrado en la transmisión o en el aprendizaje) y las prioridades de aprendizaje o la significatividad que le atribuye al aprendizaje el propio docente, además de las metodologías de evaluación que suele utilizar. Por su parte, los estudiantes que adoptan un enfoque superficial tienden a fijarse en variables más pragmáticas relacionadas con la conducta del docente: el tipo de metodología de enseñanza utilizada y los tipos de evaluación que realmente usa.

Esto sugiere que los docentes de los primeros cursos de carrera universitaria deberían utilizar tiempo y estrategias para hacer significativo el aprendizaje de sus disciplinas y cuidar el clima y la relación con sus estudiantes para ayudarles a adoptar enfoques perceptivos más amplios con los que puedan dar sentido y significado a su actividad de aprendizaje en los inicios de su carrera profesional y superar así las simples estrategias superficiales o de supervivencia en el aula.

La variable contexto de curso, entendida como los niveles de coordinación y colaboración entre las profesoras y los profesores de curso para establecer estándares comunes de carga de trabajo de los estudiantes, metodologías de enseñanza y aprendizaje parecidos, y criterios de evaluación compartidos, se ha manifestado como una variable específica de titulación que sirve para establecer diferencias entre titulaciones antiguas y de grado y entre mismas titulaciones de diferentes centros (Tabla 4). Las diferencias más significativas en la percepción del contexto de curso se dan entre los mismos estudiantes cuando están en primero o en segundo curso de carrera (Tabla 5). Estas diferencias de percepción del contexto de curso parecen ser de naturaleza distinta en cada titulación pues tienen efectos muy diversos sobre el enfoque profundo y el enfoque superficial.

La variable contexto de curso se hace perceptible de forma progresiva a los ojos de los estudiantes según van pasando cursos y condiciona sus enfoques de aprendizaje. Se hace necesario, por tanto, plantear su análisis de forma sistemática en cada curso y sugerir medidas (por ejemplo, cargas de trabajo, tipo de tareas, sistemas de evaluación coordinados, etc.) que posibiliten o no impidan el aprendizaje profundo de los estudiantes.

\section{AgradeCimientos}

Este estudio ha sido posible en el contexto del proyecto financiado por el Ministerio de Ciencia e Innovación (Micinn): Enfoques de aprendizaje de los estudiantes universitarios, metodologías docentes y contextos institucionales al inicio, intermedio y final de la carrera en el marco de los nuevos títulos. EDU2009-13195-003-03 (Subprograma EDUC). 


\section{ReferenCias Bibliográficas}

ALCOBA, J. (2012). La clasificación de los métodos de enseñanza en educación superior. Contextos educativos, 15, 93-106.

BERBEN, A.; DE LA FUENTE, J.; JUSTICIA, F. y PICHARDO, M. (2005). Análisis del aprendizaje del profesorado en formación. Revista Interuniversitaria de Formación del Profesorado, 19 (3), 225-268.

BIGGS, J. (1978). Individual and group differences in study processes. British Journal of Educational Psychology, 48, 266-279.

BIGGS, J. (1987). Student approaches to learning and studying. Hawthorn, Vic.: Australian Council for Educational Research.

BIGGS, J. (1988). The role of metacognition in enhancing learning. Australian Journal of Education, 32,127-138.

BIGGS, J. (1996). Assessing learning quality: reconciling institutional, staff and educational demands. Assessment and Evaluation in Higher education, 12 (1), 5-15.

BIGGS, J. (2001). Teaching for Quality Learning at University ( $3^{\mathrm{a}}$ ed.). Buckingham: Open University Press.

BIGGS, J.; KEMBER, D. y LEUNG, D. (2001). The revised two-factor Study Process Questionnnaire: R-SPQ-2F. British Journal of Educational Psychology, 71, 133-149.

BUENDÍA, L. y OLMEDO, E. (2000). Estrategias de aprendizaje y procesos de evaluación en la educación universitaria. Bordón, 52 (2), 151-163.

CORPAS, M. D. (2008). Estrategias de aprendizaje: la memoria en la adquisición de la lengua inglesa. Contextos educativos, 11, 23-32.

DE LA FUENTE, J.; PICHARDO, M.; JUSTICIA, F. y BERBÉN, A. (2008). Enfoques de aprendizaje, autorregulación y rendimiento académico en tres universidades europeas. Psychothema, 20 (4), 705-711.

DE LA FUENTE, J. (2011). La autoevaluación personal como determinante del enfoque de aprendizaje de la percepción del proceso de enseñanza-aprendizaje y del tipo de rendimiento. En J. Román, M. Carbonero y J. Donoso (eds.), Educación y desarrollo en una sociedad multicultural (pp. 3347-3358). Madrid: Asociación de Psicología y Educación.

ENTWISTEL, N. y RAMSDEN, P. (1983). Understanding student learning. Londres: Croom Helm.

ENTWISTLE, N.J. y TAIT, H. (1990). Approaches to learning, evaluation of teaching, and preferences for contrasting academics environments. Higher Education, 19, 169-194.

GARGAllO, B.; FERNÁNDEZ, A.; GARFELlA, P. y PÉREZ, C. (2010). Modelos de Enseñanza y Aprendizaje en la Universidad. En XXIX Seminario Interuniversitario de teoría de la Educación: Formación y Participación de los estudiantes en la Universidad. Madrid: Universidad Complutense de Madrid, noviembre 2010.

GOIKOETXEA, J.; ARAMENDI, P.; BUJÁN, K.; REKALDE, I. y ROS, I. (2010). Estudio de los contextos de enseñanza y aprendizaje universitarios percibidos por estudiantes y profesores: propuesta metodológica. En I Congreso Internacional Virtual de Formación del Profesorado. 
HIDALGO, E. (2010). Factores personales del aprendizaje: la motivación. Enfoques educativos, 58, 115-124. Disponible en: http://www.enfoqueseducativos.es/enfoques/ enfoques_58.pdf.

KEMBER, D. y GOW, L. (1994). Orientations to teaching and their effects on the quality of student learning. Journal of Higher Education, 65 (1), 59-74.

MARTON, R. y SÄLJÖ, F. (1976). On qualitative differences in learning I: Outcomes and process. British Journal of Educational Psychology, 46, 4-11.

PALAZÓN, A.; GÓMEZ-GALLEGO, M.; GÓMEZ-GALLEGO, J.C.; PÉREZ-CÁRCELES, M. C. y GÓMEZ-GARCÍA, J. (2011). Relación entre las metodologías docentes activas y el aprendizaje del estudiante universitario. Bordón, 63 (2), 27-40.

PROSSER, M. y TRIGWELL, K. (1997). Perceptions of the teaching environments and its relationships to approaches to teaching. British Journal of Educational Psychology, 67, 25-35.

PROSSER, M. y TRIGWELL, K. (1999). Understanding Learning and Teaching. The experience in Higher Education. Buckingham: Open University Press.

PROSSER, M.; TRIGWELL, K.; HAZEL, E. y GALLAGHER, P. (1994). Student's experiences of teaching and learning at the topic level. Research and Development in Higher Education, 16, 305-310.

RAMSDEN, P. (1991). A performance indicator of teaching quality in higher education: the Course Experience Questionnaire. Studies in Higher education, 16, 129150 .

RAMSDEN, P. (1992). Learning to Teach in Higher Education. London: Routledge.

RAMSDEN, P.; PROSSER, M.; TRIGWELL, K. y MARTIN, E. (1997). Perceptions of academic leadership and the effectiveness of university teaching. Annual Conference of the Australian Association for Research in Education, Brisbane, December 1997.

RAMSDEN, P. (2003). Learning to teach in higher education (2 ${ }^{\mathrm{a}}$ ed.). Nueva York: Routledge Falmer.

SAN FABIAN, J. L.; ARGOS, J. y GOIKOETXEA, J. (2009). Enfoques de aprendizaje de los estudiantes universitarios, metodologías docentes y contextos institucionales al inicio, intermedio y final de la carrera en el marco de los nuevos títulos. Proyecto de Investigación financiado por el Ministerio de Ciencia e Innovación (Micinn). EDU2009-13195-003-03 (Subprograma EDUC).

TRIGWELL, K.; PROSSER, M. y WATERHOUSE, F. (1999). Relations between teacher's approaches to teaching and student's approaches to learning. Higher Education, 37, 57-77.

VERMUNT, J. (2003). The power of learning environments and the quality of student learning. En E. de Corte, L. Verschaffel, N. Entwistle y J. Van Merriénboer (eds.), Powerful Learning Environments: Unravelling Basic Components and Dimensions. London: Pergamon y Earli.

WEINSTEIN, C. y MAYER, R. (1986). The teaching of learning strategies. En M. Wittrock (ed.), Handbook of Research of Teaching (pp. 315-327). New York: MacMillan. 
